\title{
Aprovechamiento de los residuos provenientes de cilindros y vigas de hormigón utilizados en el laboratorio de Ensayo de Materiales de la Facultad de Ingeniería, Ciencias Físicas y Matemática de la Universidad Central del Ecuador para la fabricación de bloques huecos de hormigón con limadura de acero
}

\author{
Guerra E. ${ }^{*}$; Nacata P.**; Muñoz F.*** \\ *Universidad Central del Ecuador, Facultad de Ingeniería, Ciencias Físicas y Matemática, \\ Carrera de Ingeniería Civil, Quito, Ecuador \\ e-mail: estefy-g1021@hotmail.com \\ **Universidad Central del Ecuador, Facultad de Ingeniería, Ciencias Físicas y Matemática, \\ Carrera de Ingeniería Civil, Quito, Ecuador \\ e-mail: paulync23@gmail.com \\ ***Universidad Central del Ecuador, Facultad de Ingeniería, Ciencias Físicas y Matemática, \\ Carrera de Ingeniería Civil, Quito, Ecuador \\ e-mail: fpmunoz@uce.edu.ec
}

Artículo recibido el XX de julio, 2018; revisado XX de julio de 2018. Autor para correspondencia: Freddy P. Muñoz Tobar. Correo electrónico: fpmunoz@uce, edu.ec; Teléfono: 0995822655. Quito-Ecuador

Freddy Paúl Muñoz Tobar

Docente de la Carrera de Ingeniería Civil, Facultad de Ingeniería, Ciencias Físicas y Matemática de la Universidad Central del Ecuador.

Ingeniero Civil graduado de la Carrera de Ingeniería Civil, Facultad de Ingeniería, Ciencias

Físicas y Matemática de la Universidad Central del Ecuador.

Master of Science (M.Sc.) in Municipal Water and Infrastructure specialization Drinking

Water Supply del UNESCO-IHE Institute for Water Education. Delf-The Netherlands.

\begin{abstract}
Estefanía Paola Guerra Granja. Estudiante de la Carrera de Ingeniería Civil, Facultad d Ingeniería, Ciencias Físicas y Matemática de la Universidad Central del Ecuador.

Paulina Elizabeth Ñacata Criollo. Estudiante de la Carrera de Ingeniería Civil, Facultad de Ingeniería, Ciencias Físicas y Matemática de la Universidad Central del Ecuador.

La preparación de este artículo fue posible gracias a la Carrera de Ingeniería Civil de la Universidad Central del Ecuador que permitió realizar este trabajo técnico en las instalaciones del laboratorio de Ensayo de Materiales de la Facultad de Ingeniería, Ciencias Físicas y Matemática.
\end{abstract}

Resumen

El reciclaje del hormigón en la actualidad permite generar cambios positivos en el medio ambiente toda vez que éste permite reducir la contaminación y el uso innecesario de espacios que pueden ser destinados para usos productivos. El proceso de trituración reduce los escombros de hormigón dando paso a un nuevo agregado que puede ser utilizado para distintas obras civiles con respecto a la limadura de acero, la cual, en algunos casos, es descartada sin considerar el aporte de resistencia a productos elaborados con hormigón. Los bloques huecos de hormigón fabricados a partir de estos materiales, con la dosificación óptima, lograron obtener resistencias superiores a las establecidos en la Norma Técnica Ecuatoriana NTE INEN 643 para bloque. Esta comparación se la realizó tanto para bloques fabricados de manera manual como para los fabricados de manera industrial. Su costo, en comparación con los ya existentes en el mercado, es relativamente similar. En cuanto a la resistencia a la compresión, los bloques elaborados con material reciclado poseen una resistencia superior a los bloques fabricados convencionalmente.

Palabras clave: Reciclaje de hormigón y acero, bloques, resistencia a la compresión, reducción de residuos de la construcción.

Abstract

The recycling of concrete at the moment allows to generate positive changes in the environment, since this allows to reduce the contamination and the unnecessary use of spaces that can be destined for productive uses. The crushing process reduces the concrete debris giving way to a new aggregate that can be used for different civil works with respect to steel filing, which in some cases is discarded without considering the contribution of resistance to products made with concrete. The hollow blocks of concrete manufactured from these materials, with the optimal dosage, managed to obtain resistances superior to those established in the Ecuadorian Technical Norm NTE INEN 643 for block. This comparison was made for both blocks manufactured manually and for those manufactured in an industrial manner. Its cost, compared to those already existing in the market, is relatively similar. As for the resistance to compression, blocks of recycled material have a superior resistance.

Keywords: recycling of concrete and iron, blocks, resistance to compression, reduction of construction wastes 


\section{Introducción}

Fomentar el reciclaje o reúso del hormigón en los tiempos actuales es difícil toda vez que las personas optan por botar la "basura" o escombros en lugares que no son destinados para éstos, y de esta manera se contaminan ríos y obstruyen colectores de aguas lluvia provocando inundaciones en la época invernal.

El reciclaje del concreto presenta dos ventajas principales: la primera, reduce la utilización de nuevos agregados vírgenes y los costos ambientales de explotación, transporte y asociados; y la segunda, reduce el desecho innecesario de materiales valiosos que pueden ser recuperados y reutilizados.

La falta de aprovechamiento de los residuos de los cilindros y vigas ensayados en el laboratorio de Ensayo de Materiales de la Facultad de Ingeniería, Ciencias Físicas y Matemática de la Universidad Central del Ecuador contribuye con la contaminación del medio ambiente debido a que origina focos infecciosos para la proliferación de vectores y enfermedades; además, contaminan el suelo y ocupan grandes espacios que podrían ser utilizados para otros fines.

El concreto es el segundo material más consumido después del agua y moldea la mayor parte de nuestro entorno. Viviendas, escuelas, hospitales, oficinas, vías y aceras, todos se realizan a partir del concreto. El concreto es un material durable y puede conservarse por cientos de años en muchas aplicaciones.

En el laboratorio de Ensayo de Materiales de la Facultad de Ingeniería, Ciencias Físicas y Matemática de la Universidad Central del Ecuador se realizan ensayos, tanto comerciales como académicos, por parte de los estudiantes, a los cilindros de hormigón y vigas con acero de refuerzo, los cuales posteriormente se acumulan de manera inadecuada sin ningún uso beneficioso. Mediante la potencialización de un sistema de clasificación de residuos y de un proceso adecuado para su reciclaje se pueden reutilizar los residuos como materia prima para la fabricación de nuevos materiales de construcción.

\section{Fundamentación}

\section{Componentes para los bloques de hormigón reciclado}

\section{Reciclaje de hormigón}

El concreto puede ser recuperado, triturado y reutilizado como agregado en nuevos proyectos, el cual puede conformar nuevos elementos como aceras, vías, bloques o cualquier obra que requiera agregados.

Por medio de la trituradora se logra obtener la granulometría que el constructor requiera para la realización de algún proyecto en especial.

En particular, los cilindros reciclados deben estar libres de sulfatos, plásticos, materia orgánica, vidrio, plomo y otros metales pesados. La presencia de cualquiera de los elementos antes mencionados afecta al correcto fraguado y, algunos, en interacción con el cemento, producen reacciones que afectan a la resistencia del elemento estructural, en este caso a la resistencia de los bloques de hormigón.

\section{Reciclaje de limadura de acero}

La limadura de acero tiene una alta resistencia y durabilidad, esto favorece su aplicación en la industria de la ingeniería civil.

La limadura de acero cuando se encuentra reducida a polvo es muy utilizada para mejorar el concreto de los pisos de plantas industriales, ya que éstos requieren mayor resistencia al desgaste, por lo tanto, la limadura esparcida al final de la colocación del hormigón mejora considerablemente la resistencia toda vez que ésta endura la superficie.

\section{Objetivo}

Fabricar bloques huecos con hormigón reciclado y limadura de acero que garantice resistencias superiores a las establecidas en la norma vigente.

\section{Idea a defender}

Mediante el aprovechamiento de los cilindros y vigas de hormigón ensayados, los cuales son generados en el laboratorio de Ensayo de Materia- 
les, y por medio del proceso del reciclaje, fabricar bloques huecos de hormigón con limadura de acero, dando como resultado un producto de alta resistencia.

\section{Proceso de producción}

El agregado se obtiene mediante un proceso de trituración de los cilindros, pasando por un proceso de tamizaje. El tamaño nominal del mismo será todo aquel que pase por el tamiz número \# 4 .

El cemento, que es usado para la realización de los bloques huecos de hormigón, es el Armaduro de la marca LaFarge Selva Alegre. Este cemento tiene la característica de rápido fraguado.

La limadura de acero, que fue obtenida de un taller de torno en la ciudad de Quito, fue lavada por tres días hasta que el aceite soluble, en el que se encontraba sumergida para que no se oxide, se desprenda de ésta. Es conocido que el hormigón en interacción con grasa o aceites es afectado en su adherencia con los agregados. Luego de ser lavada se la introdujo en el horno por 24 horas para separar las impurezas mediante la ayuda de un imán, aplicando el principio de electromagnetismo a fin de separar rápidamente las partículas de acero de plásticos y maderas que se encontraban presentes.

Se utilizó el método llamado densidad máxima para el diseño de la mezcla, con una relación agua/cemento de 0.49 para obtener una resistencia aproximada de 7 a $10 \mathrm{MPa}$ a los 7 días de los bloques fabricados industrialmente en la empresa "Prefabricados y Construcciones"; al mismo tiempo se realizaron bloques de manera manual con la misma relación $\mathrm{A} / \mathrm{C}$, dando como resultados resistencias de 4 a $6 \mathrm{MPa}$.

Con la finalidad de aumentar la resistencia de los bloques se añadió un 5\% de la limadura de acero con relación al peso del cemento. Este porcentaje asegura no incrementar el peso total del bloque al final del proceso.

La mezcla para la fabricación de bloques debe tener una consistencia seca, la cual se la comprueba mediante el ensayo del cono de Abrams, el cual debe tener un asentamiento igual a 0 .
No se utilizaron aditivos debido a que su uso provocaba que la mezcla se vuelva heterogénea, por lo cual se descartó su dosificación.

\section{Procesos de fabricación manual e industrial}

\section{Fabricación manual}

De acuerdo a la dosificación, acorde a los pesos exactos del agregado triturado, cemento, agua y limadura de acero, con ayuda de la concretera se obtuvo la mezcla, misma que se vertió en moldes con espesores de 10, 15 y $20 \mathrm{~cm}$. Estos moldes cumplen con las características establecidas en la norma correspondiente.

La compactación se la realizó por capas con ayuda de tacos de madera, mientras que el vibrado se realizó artesanalmente, con el uso de los combos de goma.

Una vez fabricados los bloques se los almacenó sobre una losa, dejándolos en reposo un día para que puedan ser manipulados para su respectivo curado. El curado se lo realizó humedeciéndolos con agua y luego embalándolos individualmente con papel film, y cubriéndolos con plástico negro en su totalidad.

El proceso de curado debe ser permanente, hasta un día antes del ensayo de compresión, a fin de eliminar toda presencia de humedad que afectaría en su resistencia.

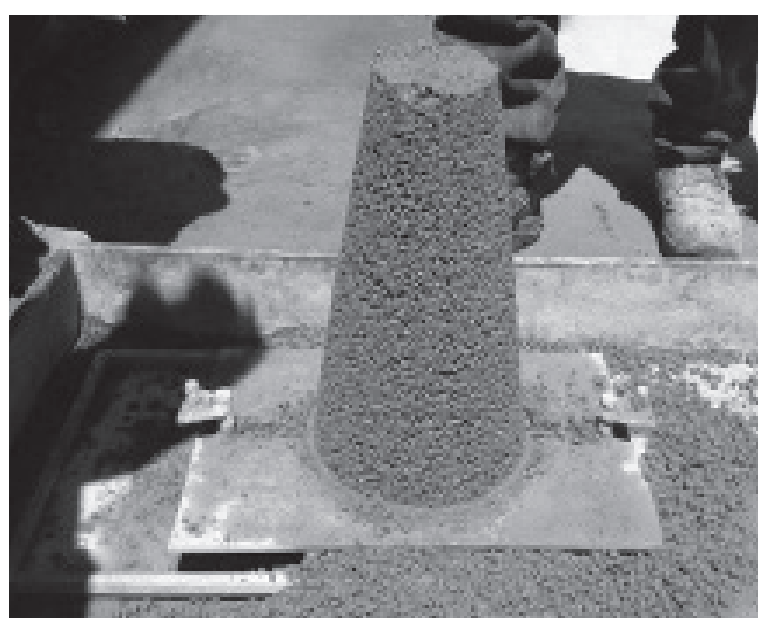

Ilustración 1. Ensayo cono de Abrams, asentamiento cero. 


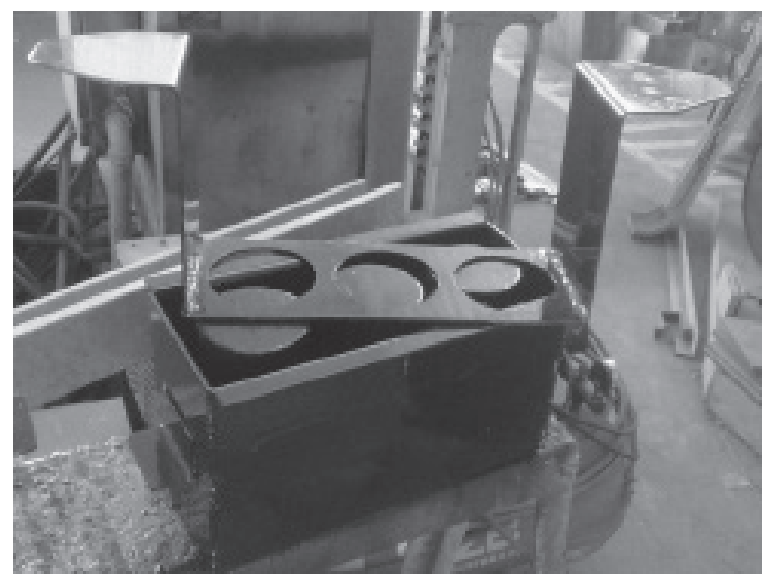

Ilustración 2. Encofrado para proceso manual, bloque de ancho $15 \mathrm{~cm}$.

\section{Fabricación industrial}

Con la misma dosificación considerada para la fabricación manual se procedió a realizar la mezcla en la empresa bloquera, la cual empieza con el mezclado y vertido en la máquina prensadora. El material, una vez colocado en los moldes, es sometido a un proceso de vibración electrónica a través de una banda vibratoria por un tiempo de un minuto y medio, la compactación de los mismos es a través de un mecanismo de prensado hidráulico.

Los bloques, una vez terminados, son dispuestos a secado natural hasta que puedan ser manipulados para luego ser sometidos al mismo proceso de curado del proceso manual.

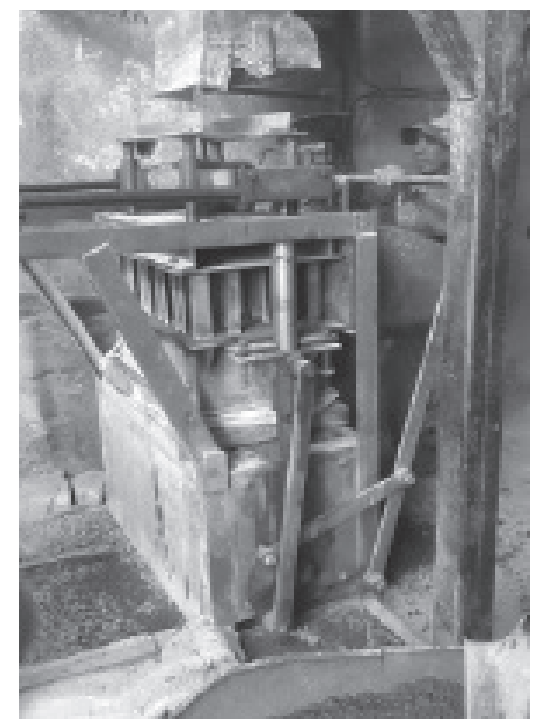

Ilustración 3. Maquina prensadora.

\section{Resultados}

Tabla 1. Peso de los bloques

\begin{tabular}{|c|c|c|}
\hline \multicolumn{3}{|c|}{ PESO BLOQUES } \\
\hline ANCHO & MANUAL & INDUSTRIAL \\
\hline $\mathrm{cm}$ & $\mathrm{kg}$ & $\mathrm{kg}$ \\
\hline \multirow{3}{*}{10} & 12.24 & 10.63 \\
\cline { 2 - 3 } & 13.85 & 10.79 \\
\cline { 2 - 3 } & 12.36 & 11.38 \\
\hline \multirow{3}{*}{15} & 10.42 & 13.18 \\
\cline { 2 - 3 } & 10.51 & 13.20 \\
\cline { 2 - 3 } & 10.87 & 13.15 \\
\hline \multirow{3}{*}{20} & 14.44 & - \\
\cline { 2 - 3 } & 14.73 & - \\
\cline { 2 - 3 } & 15.02 & - \\
\hline
\end{tabular}

Tabla 2. Resultados del ensayo de compresión

\begin{tabular}{|c|c|c|}
\hline \multicolumn{3}{|c|}{ ENSAYO DE COMPRESIÓN NE BLOQUES } \\
\hline ANCHO & MANUAL & INDUSTRIAL \\
\hline $\mathrm{cm}$ & $\mathrm{MPa}$ & $\mathrm{MPa}$ \\
\hline \multirow{3}{*}{10} & 5.15 & 6.45 \\
\hline & 2.78 & 6.84 \\
\hline & 7.87 & 7.55 \\
\hline$\overline{\text { PROMEDIO }}$ & 5.27 & 6.95 \\
\hline \multirow{3}{*}{15} & 4.72 & 9.00 \\
\hline & 5.83 & 11.86 \\
\hline & 7.02 & 9.72 \\
\hline PROMEDIO & 5.86 & 10.19 \\
\hline \multirow{3}{*}{20} & 4.57 & - \\
\hline & 4.58 & - \\
\hline & 4.27 & - \\
\hline PROMEDIO & 4.47 & - \\
\hline
\end{tabular}

\section{Discusión}

La fabricación de los bloques se puede realizar de forma manual o industrial en virtud de que los resultados obtenidos en los ensayos de compresión en laboratorio comparados con la norma NTE INEN 643 son óptimos para que el producto sea comercializado a los 7 días de edad, consideran- 
do principalmente que los bloques son elementos que deben tener condiciones de rápido fraguado, alcanzando resistencias esperadas en los primeros días.

El precio unitario de los bloques está dispuesto en función de la cantidad de materia prima y recursos utilizados para su fabricación. Como es el caso del cemento, el cual tiene el mayor grado de importancia para obtener una mayor resistencia. Aquí también se debe considerar el costo de otros recursos indispensables como son: maquinaria, mano de obra y costo de servicios básicos necesarios para conseguir el producto final.

El impacto ambiental que se genera al fabricar los bloques bajo este principio es positivo porque reduce la producción de escombros mediante el reciclaje. Además, para aumentar la producción de los bloques se necesitaría dos condicionantes: (1) acopiar un volumen de escombros sin material orgánico en el laboratorio, y (2) la donación de limadura de acero por parte de empresas comercializadoras de este producto como Novacero y Adelca.

Estos bloques pueden destinarse para ser usados en muros simples o estructurales, ornamentales, primeras plantas de edificaciones, paredes divisorias internas de las construcciones, muros de contención, entre otros.

\section{Conclusiones}

- Se limita su uso para obras en los que no se empleen como elementos de alivianamiento dado su mayor peso en comparación a los bloques de fabricación convencional.

- La compresión de los bloques no se puede realizar a los 28 días debido a la necesidad inmediata de comercialización, por lo cual la resistencia esperada se debe obtener en los 7 primeros días.

- Según la norma NTE INEN 643 la resistencia mayor es de $6 \mathrm{MPa}$ a los 28 días para un bloque tipo A; a su vez los bloques fabricados manualmente alcanzan una resistencia similar en un menor tiempo.

- Los resultados de compresión presentan rela- ción directa en función del procedimiento de curado de los bloques.

- Los bloques industrializados fabricados con la dosificación óptima superan en un 40\% la mayor resistencia según la norma NTE INEN 643.

- Un impacto ambiental negativo generado es la producción de polvo y ruido producido por la maquinaria durante la trituración.

- El rango de precios de un bloque de hormigón fabricado bajo esta modalidad va desde los USD 0.55 hasta USD 1.20 dependiendo de las dimensiones. Para un bloque de espesor de $10 \mathrm{~cm}$ el PVP es de USD 0.75; mientras que para un bloque de espesor de $15 \mathrm{~cm}$ el PVP es de USD 1.30.

\section{Referencias}

[1] Instituto Ecuatoriano de Normalización. (2012). INEN 643. Obtenido de http:// normaspdf.inen.gob.ec/pdf/nte/639-2. pdf

[2] Iniciativa por la sostenibilidad del cemento. (Julio de 2009). Reciclando concreto. Obtenido de ficem: http://ficem. org/publicaciones-CSI/DOCUMENTO-CSI-RECICLAJE-DEL-CONCRETO/RECICLAJE-D-CONCRETO_1.pdf

[3] Maza, C. L. (2007). repositorio.uchile. Obtenido de http://repositorio.uchile.cl/ bitstream/handle/2250/120397/Evaluacion_de_Impactos_Ambientales.pdf

\section{Agradecimientos}

- Guerra E.P. Agradezco a mi familia, amigos y Jonathan por su apoyo incondicional en todo momento. A mi compañera de fórmula Pauly con quien pude hacer realidad este sueño, al laboratorio de Ensayo de Materiales por colaborarnos, y en especial al ingeniero Freddy Muñoz por creer en este proyecto y apoyarnos para hacerlo realidad. 
- Ñacata P.E. Agradezco a mi familia, amigos y todas las personas que colaboraron en la realización de esta meta, al laboratorio de Ensayo de Materiales por ayudarnos a realizar nuestra tesis. A mi compañera de tesis
Estefanía por culminar este sueño juntas, y en especial al ingeniero Freddy Muñoz por creer en este proyecto y apoyarnos para hacerlo realidad. 